\title{
Ependymoma, RELA Fusion-Positive
}

National Cancer Institute

\section{Source}

National Cancer Institute. Ependymoma, RELA Fusion-Positive. NCI Thesaurus. Code C129351.

An ependymoma characterized by gene fusions involving RELA, a transcription factor involved in NK-kB pathway activity. It accounts for the majority of supratentorial ependymomas in children. It has an unfavorable outcome when compared to other ependymoma subtypes. 